\title{
Local scaling property of seismicity: an example of getting valuable information from complex hierarchical system
}

\author{
Q. Li and G.-M. Xu \\ Research Center for Earthquake Prediction, Earthquake Administration of Jiangsu Province, Nanjing 210014, China
}

Received: 18 January 2010 - Revised: 25 July 2010 - Accepted: 26 July 2010 - Published: 10 September 2010

\begin{abstract}
In order to get valuable information about the scale invariance in the process of seismogeny of Lijiang $M 7.0$ earthquake, the scaling property of the interevent time series of the seismic sequences for Lijiang area in China were studied by using the method of the local scaling property, the generalised dimension spectrum, and the correlation dimension. It is found that there is a clear characteristic variation of local scaling property prior to Lijiang $M 7.0$ earthquake while there is no characteristic variation of the generalised dimension spectrum and the correlation dimension. The reason for producing this phenomenon is that the fractal seismic system is a complex hierarchical system. For such a system, searching for a relevant choice in application of the three methodologies is needed. Compared with the generalised dimension spectrum and mono-fractal dimension which focus on the global description of the scaling properties of fractal objects, the local scaling property emphasizes the local features, and can give the local information of the singularity of the fractal system, therefore, it is easier for us to get valuable information from complex hierarchical structure with this method.
\end{abstract}

\section{Introduction}

Recently, more and more attention was focused on the study of the nonlinear characteristics of seismicity and seismogeny. It has been shown in rock burst (Lu et al., 2005), petrophysical experiments (Lei et al., 2003; Lei and Satoh, 2007) and natural earthquake research (Murase, 2004; Roy and Nath, 2007) that the mono-fractal dimension displays characteristic changes before a major rupture; it has also been shown in multifractal research on seismic activity that the spectrum of generalised dimensions and the singularity spectrum display characteristic changes before large earthquakes (Caruso et al., 2006; Dimitriu et al., 2000; Nakaya, 2005; Roy and Padhi, 2007). Some results have confirmed that the decrease in fractal dimension, the steepening of curves in the generalised dimension spectrum and the broadening of the value range in the singularity spectrum, can be taken as indicators of an unstable state of an earthquake system before a large rupture (Carpinteri et al., 2009; Goltz, 1997; Kiyashchenko et al., 2004; Lee et al., 2008; Matcharashvili et al., 2000; Radulian and Trifu, 1991; Telesca et al., 2005; Telesca and Lapenna, 2006). However, there are some shortcomings in the research: the mono-fractal dimension is not sufficient to characterise heterogeneous fractals (Grassberger and Procaccia, 1984; Kentz and Schreiber, 1997), while the generalised dimension spectrum and singularity spectrum can only represent a global description of the scaling properties (Chhabra and Jensen, 1989; Grassberger, 1983). As a result, there is the possibility of losing valuable information on the fractal properties and the universality of such research results is in dispute.

On the other hand, the fractal seismic system is a complex system (Chelidze and Matcharashvili, 2007; Kagan, 1994; Keilis-Borok, 1990), and it is hierarchical in structure. In order to get valuable information about scaling property from this hierarchical structure, we use the method of local scaling property in addition to the method of the generalised dimension spectrum and the correlation dimension. Because the local scaling property can help us to analyse the minute local features of a fractal, obtaining local information of the singularity of the fractal system makes this meaningful.

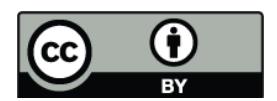

Correspondence to: $\mathrm{Q}$. $\mathrm{Li}$

(lqdzjybzx@126.com)

Published by Copernicus Publications on behalf of the European Geosciences Union and the American Geophysical Union. 


\section{Method}

\subsection{Local scaling property}

The method of local scaling property is as follows (Arneodo et al., 1988; Liu and Cheng, 1999; Yang, 2003).

A typical property of fractals is that they are asymptotically self-similar at small length scales. Let us consider a fractal represented by a real function $f$. Looking near an arbitrary point $x_{0}$ at different scales, we always find the same function up to a scaling factor. Defining

$f_{x_{0}}(x)=f\left(x_{0}+x\right)-f\left(x_{0}\right)$, then we have

$f_{x_{0}}(\lambda x)=\lambda^{+\alpha\left(x_{0}\right)} f_{x_{0}}(x)$

Here, $\alpha\left(x_{0}\right)$ is the local scaling exponent (also called the singularity exponent or Hölder exponent), representing the singularity strength at position $x_{0}$.

It can be proven that the wavelet transform coefficient of $f(x)$ around $x_{0}$ also displays a similar property of scale invariance. Define

$T\left(a, x_{0}+b\right)=\frac{1}{\sqrt{a}} \int f(x) \psi\left(\frac{x-x_{0}-b}{a}\right) d x$

as the wavelet transform around $x_{0}$, and

$T\left(\lambda a, x_{0}+\lambda b\right)=\frac{1}{\sqrt{\lambda a}} \int f(x) \psi\left(\frac{x-x_{0}-\lambda b}{\lambda a}\right) d x$

as the form of the wavelet transform coefficient around $x_{0}$ when the scale varies. This transformation can be seen as a mathematical microscope whose position and magnification are $b$ and $1 / a$, respectively, and whose optics are given by the choice of the specific wavelet $\psi$. Then, we have

$T\left(\lambda a, x_{0}+\lambda b\right)=\lambda^{+\alpha\left(x_{0}\right)+\frac{1}{2}} T\left(a, x_{0}+b\right)$

Therefore, based on the Eq. (2), we can obtain local scaling exponent $\alpha$ of the fractal objects by analysing the scaling property of the wavelet transform $T(a, b)$. In actual application of the case, we can study the local scaling property of the fractal by transforming the parameters $1 / a$ and $b$. For example, we can observe the variation of the transform $T(a, b)$ with the magnification $1 / a$ by plotting $\ln |T(a, b)|$ versus $\ln a$ with a fixing $b$. For point $b$ at different positions where there is asymptotic self-similarity at small length scales, the plot of $\ln |T(a, b)|$ versus $\ln a$ is a straight line with slope $K$. The local scaling exponent $\alpha$ at that point can be easily calculated from the following equation:

$K=\alpha+\frac{1}{2}$

In our study, we need to know the local scaling exponent $\alpha$ of every point in series. Through the change of parameter $b$, we can obtain the local scaling exponent $\alpha$ at each point.

\subsection{The generalised dimension spectrum $D_{\mathrm{q}}$ and correlation dimensions $\mathrm{D}_{2}$}

The generalised dimension spectrum $D_{q}$ is analysed by a method based on the correlation integral (Grassberger, 1983). The calculation of $D_{q}$ using the correlation integral method (Nakaya, 2005) is performed using

$D_{q}=\lim _{r \rightarrow 0} \log C_{q}(r) / \log r$,

where $C_{q}(r)$ is the $q$ th-order generalised correlation function, given as

$C_{q}(r)$

$=\left\{\frac{1}{N} \sum_{j=1}^{N}\left[(1 /(N-1)) \sum_{\substack{i=1 \\ i \neq j}}^{N} \Theta\left(r-\left|X_{i}-X_{j}\right|\right)\right]^{q-1}\right\}^{\frac{1}{q-1}}$

with $r$ as the scaling radius, $\Theta(s)$ as the Heaviside step function, $N$ as the number of data points and $\left|X_{i}-X_{j}\right|$ as the distance between the two points $X_{i}$ and $X_{j}$. If the series has a fractal structure, then $C_{q}(r)$ is represented by a power law. The $D_{q}$ value is determined by the slope of the linear segment in the graph of $\log C_{q}(r)$ versus $\log r$.

As for the correlation dimension $D_{2}$, it is the special case of generalised dimension. Letting $q=2$ in Eqs. (4) and (5), we can easily get $D_{2}$.

\section{Data}

The data consist of interevent times between successive earthquakes exceeding a threshold magnitude. Our choice was made by considering that the earthquake occurrence time is one of the most reliable and accurate parameters that define a seismic event (Enescu et al., 2006; Telesca et al., 2004). Also, our choice was based on the correlation of earthquake recurrence times.

The data adopted are from a very seismically active area of Lijiang in China. The area around Lijiang was struck by a violent earthquake $(M=7.0)$ on 3 February 1996. The epicentre distribution of events occurred from 1984 to 2000 in the Lijiang area (total 2296 earthquakes) is shown in Fig. 1 (data extracted from the earthquake catalogue of the China Earthquake Administration). The earthquakes are located in a circular area centred on the epicentre of the above violent earthquake, with a radius of about $160 \mathrm{~km}$. The interevent time series of the seismicity with a logarithmic scale is shown in Fig. 2 (the order number of the main shock is 1601). The completeness magnitude estimated after performing Gutenberg-Richter analysis is 2.4 (see Fig. 3). 


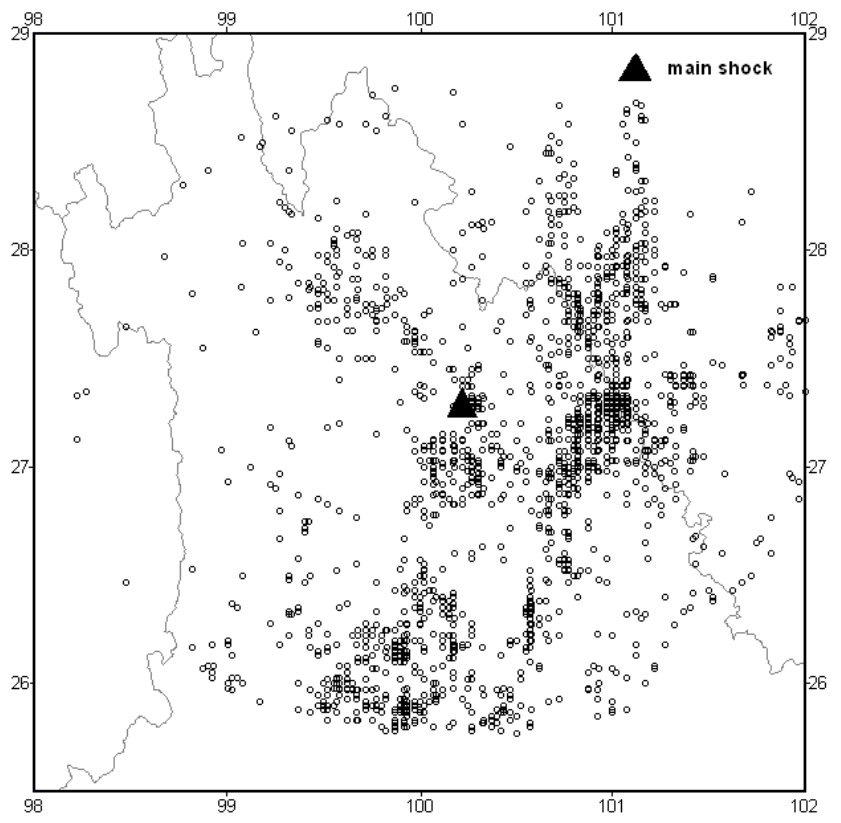

Fig. 1. Epicentre distribution of the seismicity in Lijiang area during the period 1984-2000.

\section{Results and analysis}

\subsection{Local scaling property}

On the basis of Eq. (2), the $\ln |T(a, b)|$ versus $\ln a$ plot with different positions $b$ can be obtained by using the Mexican hat wavelet. The plots are two different types: one approximates a straight line while the other fluctuates considerably. For an approximate straight line, we can get the slope $K$ by using a least squares fit with a check value $(F=21$ for a F-test at 99\% confidence level), thus, we can calculate the local scaling exponent $\alpha$ at that point from Eq. (3), which means that the interevent time series is singular at that point (we call this point a singular point). For a "non-straight line", we can get neither the slope $K$ through fitting nor the local scaling exponent $\alpha$, which means that the series is not singular at that point. As a result, some points in the series are singular while others are not.

Figure 4 shows the local scaling exponent $\alpha$ versus the point $n$ of the series: points with missing data in the abscissa represent that the series are not singular at these points. We note that singular points are dense in some periods of the series while sparse in others, which means that the distribution of singular points at different seismogenic stages displays different characteristics.

By using the concept of a sliding window (Telesca and Lapenna, 2006), one can calculate the temporal evolution of the distribution characteristics of singular points. In order to evaluate the calculation results with one standard, we used

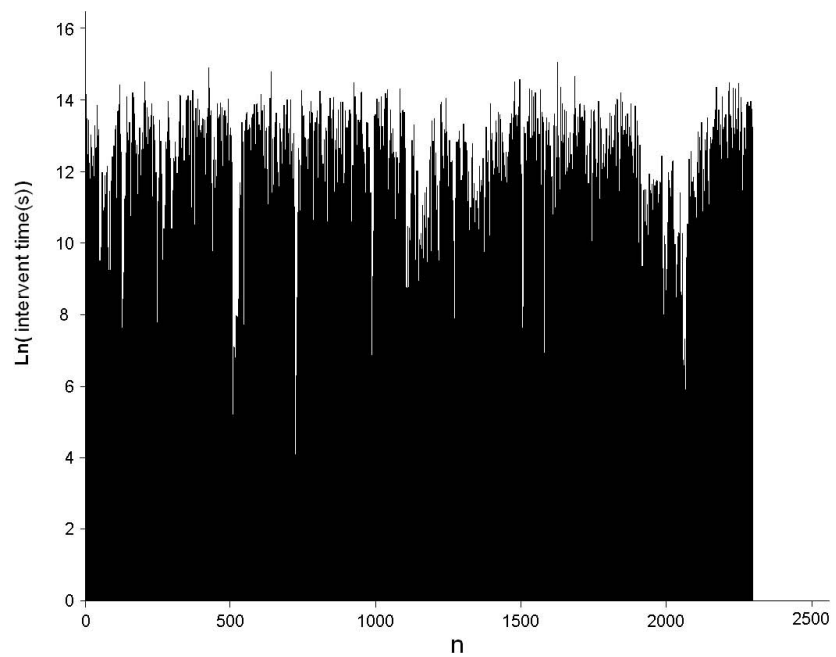

Fig. 2. The interevent interval time series of the seismicity shown in Fig. 1 with logarithmic scale.

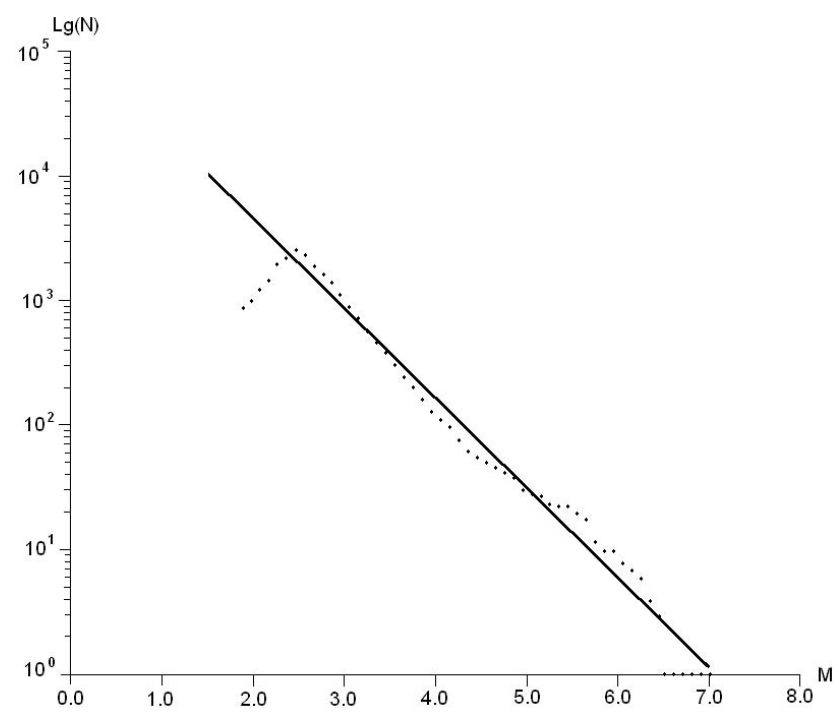

Fig. 3. Magnitude-frequency relationship of the seismicity in Lijiang area during the period 1984-2000.

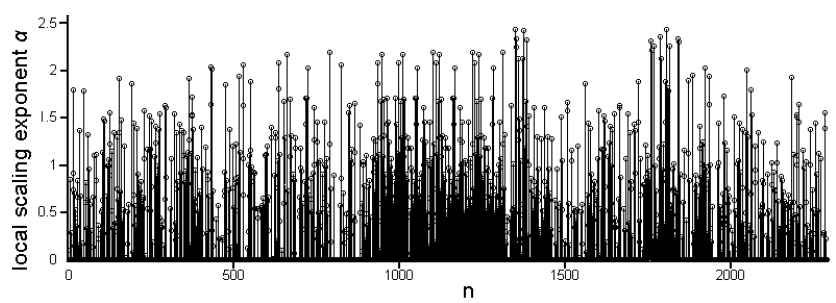

Fig. 4. The local scaling exponent $\alpha$ versus point $n$ of the series. Points with missing data in the abscissa represent that the series are not singular at these points. 


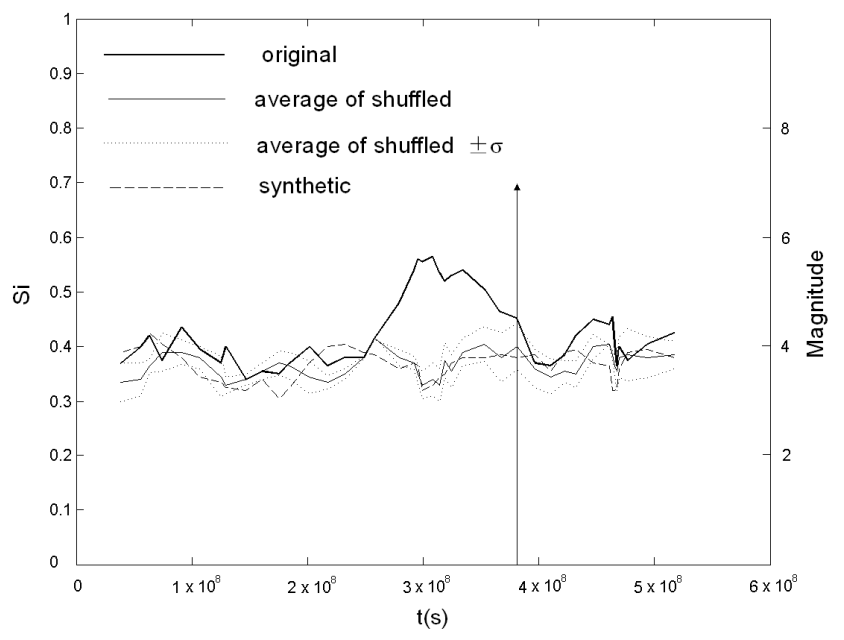

Fig. 5. The variation of $S i$ versus time. In the figure, the $S i$ value for the original series (thick solid line), the average (fine solid line) of the $S i$ value obtained by 100 randomly shuffled versions within their $\pm 1 \sigma$ range (fine dotted lines) and the $S i$ value obtained by synthetic data(fine dashed line) are plotted. The vertical arrow indicates $M 7.0$ Lijiang earthquake.

the same sliding window and the same shift between two successive windows in the calculation made by using the three above-mentioned methods. In our case, we considered a sliding window of 200 events (the average duration of the sliding window of 200 events for a point in time scale is 17.7 months, and the duration prior to the main shock is 21 months). The shift between two successive windows was set to 50 events, in order to smooth the results and evaluate the variation of the distribution characteristics of singular points with good time resolution.

Next, we define parameter $\mathrm{Si}$ as the number of singular points per unit sliding window length (i.e. $S i=N / L$ ). As a result, high numerical value of $S i$ signifies a dense distribution of singular points, while low numerical value signifies a sparse distribution of singular points.

The time variation of $\mathrm{Si}$ for the series is shown in Fig. 5 (thick solid line), and is obtained using the sliding window method(the point of each sliding window is on the right side), based on Fig. 4.

The most striking feature in the plot is the significant change in parameters $S i$ before the main shock. The parameter shows an increase before the main shock, which means that within the sliding window, the number of singular points is larger than during normal periods. After the main shock, the parameter shows a decrease, which means that within the sliding window, the number of singular points becomes smaller again. This result indicates that there is a clear characteristic variation of local scaling property prior to Lijiang $M 7.0$ earthquake.
In order to verify that the variability of the parameter is not random but significant, we performed the same analysis on 100 randomly shuffled versions of the original series. We calculated for each shuffled series the $S i$ value varying with time, and then we averaged them. In Fig. 5 we also plotted the average (fine solid lines) within their $\pm 1 \sigma$ range (fine dotted lines; $1 \sigma$ is called standard error). We can clearly observe that the $S i$ value calculated for the original series differs significantly from those calculated for the shuffled series: the $S i$ value for the shuffled series has fluctuations in a small range but has no characteristic variation in different periods of time.

we know that usually a certain percentage of the catalog consists of aftershocks triggered by larger magnitude events with an Omori-type decay in time. Therefore, aftershocks might dominate any measure applied to seismicity data. In order to verify that the aftershocks are not responsible for the drop of Si after the main shock, the synthetic data consisting of Poissonian background events plus aftershock sequences (i.e. by applying the ETAS model by Ogata (1988, 1989)) are tested. According to the research on seismicity and the features of ETAS model parameters in Lijiang area of China (Jiang et al., 2007), we chose Poisson rate $\lambda=135$ (an average of 135 earthquakes of $M_{1} \geq 2.4$ per year as Poissonian background events) and the suitable ETAS model parameters (the decay ratio of the aftershock $p=0.778$, frequency-magnitude distribution $b=0.721$, the ability of sequences to generate high order aftershocks $\beta=0.993$ ) for the test. We calculated for the synthetic data the $S i$ value varying with time. In Fig. 5, we plotted the variation of $S i$ value for the synthetic data (fine dashed lines). We can observe that the aftershock sequence is not responsible for the drop of $S i$ after the main shock.

Consequently, the above mentioned characteristic variation of local scaling property indicates a new mechanism that traditional linear theory can not explain. According to complex system theory (Xu, 2000), a system is composed of many components. The components in a linear system are independent. However, the components in a nonlinear system are interdependent and interactive, so an increase in the number of components will enhance the interaction among components, thus, increasing the instability of the system. In our nonlinear system of seismic activity (interevent time series), the singular points are considered to be the components of the fractal system. In this sense, a different number of components (i.e. singular points) can be attributed to the difference in system properties. Before the main shock, the increasing number of singular points (i.e. the increasing number of components) enhances their interaction, thus, increasing the instability of the seismic system. In normal times and after the main shock, within the same window length, the number of singular points remains at a smaller value, showing that the system is in a state of stability. 


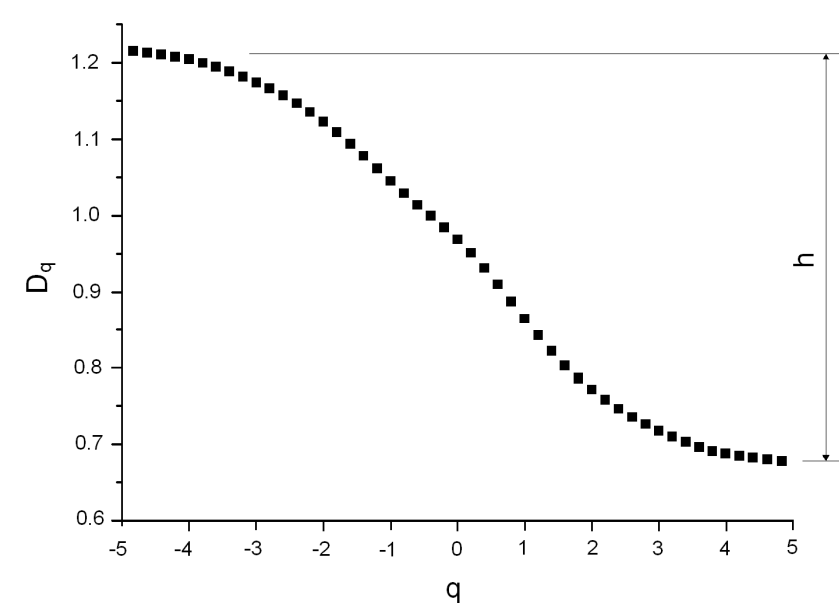

Fig. 6. The generalised dimension spectrum $D_{q}$ for $q$ ranging between -5 and 5 .

\subsection{Generalized dimension spectrum and correlation dimension}

Figure 6 shows the variation of the generalised dimension spectrum of seismic series relevant to the case of seismic interevent time series. The curve presents that $D_{q}$ for positive $q$ decreases as $q$ is increased and for negative $q$ increases as $q$ is decreased, which illustrates the multifractal property. Some researches show that the $D_{q}-q$ curves become steeper before a great rupture than during normal periods (Hirabayashi et al., 1992; Nakaya, 2005; Zhu and Chen, 2000), i.e., $D_{q}$ increases for negative $q$ and decreases for positive $q$. This change mainly occurs within the range of $-5 \leq q \leq 5$.

In order to describe the variation of the generalised dimension spectrum mentioned above quantitatively, we define $h=D_{q=-5}-D_{q=5}$, which we use for the characteristic parameter of the variation of the generalised dimension spectrum. Besides, in order to show this variation before and after Lijiang $M 7.0$ earthquake, the concept of a sliding window is used. We calculated the interevent time series of the seismic sequences for Lijiang area. Figure 7 shows the parameter $h$ versus the time. The plot presents the parameter $h$ has fluctuations but has no characteristic variation in different periods of time, which indicates that the generalised dimension spectrum has no characteristic variation before Lijiang $M 7.0$ earthquake. Therefore, we can not get any valuable result from this analysis.

Now, let us see the result of fractal dimension. Some researches show the decrease of the fractal dimensions before a great rupture (Carpinteri et al., 2009; Goltz, 1997; Kiyashchenko et al., 2004; Lee et al., 2008; Matcharashvili et al., 2000; Radulian and Trifu, 1991). Because the

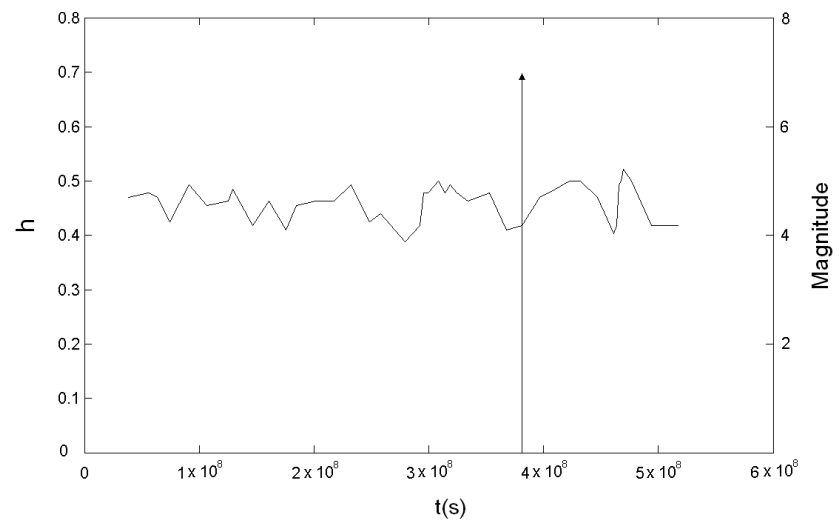

Fig. 7. The variation of $h$ versus time. The vertical arrow indicates M 7.0 Lijiang earthquake.

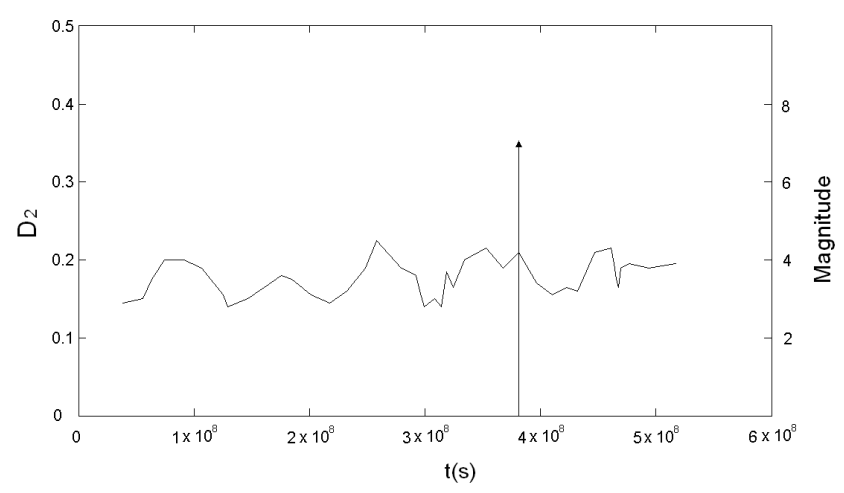

Fig. 8. The variation of correlation dimension $D_{2}$ versus time. The vertical arrow indicates $M 7.0$ Lijiang earthquake.

correlation dimension $D_{2}$ is the most typical in the fractal dimensions, we only research $D_{2}$ in this paper. In order to show the variation of $D_{2}$ with the time, the concept of a sliding window (Martin et al., 2000) is also used. We calculated the interevent time series. Figure 8 shows the variation of $D_{2}$ versus the time. The plot presents the parameter $D_{2}$ has fluctuations but has no characteristic variation in different periods of time.

From the above study, we have the valuable information about the scale invariance in the seismogenic process of Lijiang $M 7.0$ earthquake with the method of local scaling property, however, we can not get any valuable information with the method of generalised dimension spectrum and correlation dimension. The reason for producing this phenomenon is related to the complexity of the fractal seismic system. 


\section{Discussion and conclusion}

Our research on the scaling property of seismicity has demonstrated that there is a clear characteristic variation of local scaling property prior to Lijiang $M 7.0$ earthquake while there is no characteristic variation of the generalised dimension spectrum and the correlation dimension. In fact, a complex fractal system is hierarchical in structure (Badii and Politi, 1999). It is composed of some components and they are composed of some lower level components. Different levels of components have their own local information. However, the global description of the scaling properties neglects this structure, and produces an effect of "smoothing" of information. This effect of "smoothing" can result in the loss of valuable local information. Compared with the global description of the scaling properties (generalised dimension spectrum and mono-fractal dimension), the local scaling property emphasizes the local features, and can give the local information of the singularity of the fractal system, therefore, it is easier for us to get valuable information from complex hierarchical structure with this method.

Besides, as can be seen from the above analysis, the choice of suitable methods is needed for the purpose of getting valuable information in application of the three methodologies. If we have no search for a relevant choice, we might not get any valuable result from this analysis.

Acknowledgements. We thank S. S. Dong for helpful conversations and D. Cushley for critical reviews. This work is supported by the Natural Science Foundation of Jiangsu Province, China (BK2008486).

Edited by: J. Davidsen

Reviewed by: V. Kossobokov and two other anonymous referees

\section{References}

Arneodo, A., Grasseau, G., and Holschneider, M.: Wavelet Transform of Multifractals, Phys. Rev. Lett., 61, 2881-2884,1988.

Badii, R. and Politi, A.: Complexity: hierarchical structures and scaling in physics, Cambridge University Press, Cambridge, UK, 1999.

Carpinteri, A., Lacidogna, G., and Puzzi, S.: From criticality to final collapse: Evolution of the "b-value" from 1.5 to 1.0, Chaos, Soliton. Fract., 41, 843-853,2009.

Caruso, F., Vinciguerra, S., Latora, V., Rapisarda, A., and Malone, S.: Multifractal analysis of Mount St. Helens seismicity as a tool for identifying eruptive activity, Fractals, 14, 179-186, 2006.

Chelidze, T. and Matcharashvili, T.: Complexity of seismic process; measuring and applications - A review, Tectonophysics, 431, 49-60, 2007.

Chhabra, A. and Jensen, R. V.: Direct determination of the $\mathrm{f}(\alpha)$ singularity spectrum, Phys. Rev. Lett., 62, 1327-1330, 1989.
Dimitriu, P. P., Scordilis, E. M., and Karacostas, V. G.: Multifractal Analysis of the Arnea, Greece Seismicity with Potential Implications for Earthquake Prediction, Nat. Hazards, 21, $277-$ 295, 2000.

Enescu, B., Ito, K., and Struzik, Z. R.: Wavelet-based multiscale resolution analysis of real and simulated time-series of earthquakes, Geophys. J. Int, 164, 63-74, 2006.

Goltz, C.: Fractal and Chaotic Properties of Earthquakes, SpringerVerlag, Berlin, 1997.

Grassberger, P.: Generalized Dimensions of Strange Attractors, Phys. Lett., 97A, 227-320,1983.

Grassberger, P. and Procaccia, J.: Dimensions and Entropies of Strange Attractors from A Fluctuating Dynamics Approach, Physica D, 13, 34-54, 1984.

Hirabayashi, T., Ito, K., and Yoshii, T.: Multifractal analysis of earthquakes, Pure Appl. Geophys., 138, 592-610, 1992.

Jiang, H. K., Zheng, J. C., Wu, Q., Qu, Y. J., and Li, Y. L.: Earlier statistical features of ETAS model parameters and their seismological meanings, Chinese J. Geophys., 50, 1778-1786, 2007 (in Chinese).

Kagan, Y. Y.: Observational evidence for earthquakes as a nonlinear dynamic process, Physica D, 77, 160-192, 1994

Keilis-Borok, V. I.: The lithosphere of the Earth as a nonlinear system with implications for earthquake prediction, Rev. Geophys., 28, 9-34, 1990.

Kentz, H. and Schreiber, T.: Nonlinear Time Series Analysis, Cambridge University Press, Cambridge, UK, 1997.

Kiyashchenko, D., Smirnova, N., Troyan, V., Saenger, E., and Vallianatos, F.: Seismic hazard precursory evolution: fractal and multifractal aspects, Phys. Chem. Earth, 29, 367-378, 2004.

Lee, Y. T., Chen, C. C., Chang, Y. F., and Chiao, L. Y.: Precursory phenomena associated with large avalanches in the long-range connective sandpile (LRCS) model, Physica A, 387, 5263-5270, 2008.

Lei, X. L., Kusunose, K., Satoh, T., and Nishizawa, O.: The hierarchical rupture process of a fault: an experimental study, Phys. Earth Planet. In., 137, 213-228, 2003.

Lei, X. L. and Satoh, T.: Indicators of critical point behavior prior to rock failure inferred from pre-failure damage, Tectonophysics, 431, 97-111, 2007.

Liu, F. and Cheng, J. Z.: Local Fractal Scale Wavelet Analysis, Journal of Xi'an Jiaotong University, 33, 14-34, 1999 (in Chinese).

Lu, C., Mai, Y. W., and Xie, H.: A sudden drop of fractal dimension: a likely precursor of catastrophic failure in disordered media, Phil. Mag. Lett., 85, 33-40, 2005.

Martin, M. T., Plastino, A. R., and Plastino, A.: Tsallis-like information measures and the analysis of complex signals, Physica A, 275, 262-271, 2000.

Matcharashvili, T., Chelidze, T., and Javakhishvili, Z.: Nonlinear analysis of magnitude and interevent time interval sequences for earthquakes of the Caucasian region, Nonlin. Processes Geophys., 7, 9-20, doi:10.5194/npg-7-9-2000, 2000.

Murase, K.: A Characteristic Change in Fractal Dimension Prior to the 2003 Tokachi-oki Earthquake (MJ = 8.0), Hokkaido, Northern Japan, Earth Planets Space, 56, 401-405, 2004.

Nakaya, S.: Fractal properties of seismicity in regions affected by large, shallow earthquakes in western Japan: Implications for fault formation processes based on a binary fractal 
fracture network model, J. Geophys. Res., 110, B01310, doi:10.1029/2004JB003097, 2005.

Ogata, Y.: Statistical model for earthquake occurrence and residual analysis for point processes, J. Am. Stat. Assoc., 17, 97-105, 1988.

Ogata, Y.: Statistical model for standard seismicity and detection of anomalies by residual analysis for point processes, Tectonophysics, 83, 9-27, 1989.

Radulian, M. and Trifu, C.-I.: Would It Have Been Possible to Predict the 30 August 1986 Vrancea earthquake?, B. Seismol. Soc. Am., 81, 2498-2503, 1991.

Roy, P. N. S. and Nath, S. K.: Precursory correlation dimensions for three great earthquakes, Current Science, 93, 1522-1529, 2007.

Roy, P. N. S. and Padhi, A.: Multifractal analysis of earthquakes in the southeastern Iran-bam region, Pure Appl. Geophys., 164, 2271-2290, 2007.
Telesca, L., Lapenna, V., and Macchiato, M.: Mono- and multifractal investigation of scaling properties in temporal patterns of seismic sequences, Chaos Soliton. Fract., 19, 1-15, 2004.

Telesca, L., Lapenna, V., and Macchiato, M.: Multifractal fluctuations in seismic interspike series, Physica A, 354, 629640, 2005.

Telesca, L. and Lapenna, V.: Measuring multifractality in seismic sequences, Tectonophysics, 423, 115-123, 2006.

Xu, Z. G.: Systems Science, Shanghai Scientific \&Technological Education Publishing House, Shanghai, 2000 (in Chinese).

Yang, F. S.: Application of Wavelet Transform on engineering analysis, Science Press, Beijing, 2003 (in Chinese).

Zhu, L. R. and Chen, R.: Fractal in earthquakes, Seismological Press, Beijing, 2000 (in Chinese). 